Book Review

DOI: $10.1038 / \mathrm{sj} / \mathrm{sc} / 3101294$

\section{Neuroanatomy and Neurophysiology}

JS Citow, RL Macdonald

Published by Thieme Medical Publishers Inc.: New York, 181 pp. ISBN 313129 2210. DEM 179

\section{Neuropathology and Neuroradiology}

JS Citow, RL Wollmann, RL Macdonald

Published by Thieme Medical Publishers Inc.: New York, 239 pp. ISBN 313129 2318. DEM 179

These two companion volumes are welcome additions to the body of literature available for those training in the neurosciences. They represent a valiant attempt by the authors to classify and summarise material pertinent to the fields described in the titles. To present these in a review format necessitates that most areas can only be covered with broad brush strokes. Emphasis is given to those subjects most likely to be of importance to neurosurgeons, unsurprisingly since the author is from this field.

The Neuroanatomy section is concise yet contains more than sufficient textual detail for most purposes. The illustrations are a mixture of new drawings, those drawn from standard texts and radiographs. With such a great amount of detailed information, the importance of sufficient, clear illustrations cannot be underestimated and I think that this section perhaps could benefit from more carefully thought out diagrammatic support to lift it to the highest standard.

The Neurophysiology section encompasses both useful reminders of basic concepts and quite detailed descriptions of neurophysiological mechanisms. In some instances, the text could have struck a better balance between (near useless) brevity and endless detail about relatively trivial areas. There are some small errors, inconsistencies and omissions, particularly noticeable when referring to current clinical neurological practice. However, the scope of the chapter is vast and does attempt to present the information in a readable and interesting manner.

Combining Neuropathology and Neuroradiology into an integrated format worked well and is the stronger of the two volumes. The content is organised by disease category and provides an accessible overview of each area. The section on tumours of the nervous system is especially well-presented and is supported by generous numbers of illustrations. This emphasis again favours neurosurgeons. The description of those diseases usually in the realm of the neurologist can be rather basic and oversimplified. In some instances, facts are given in what appears a somewhat random manner. For example, the subsection on muscle disease provides little flavour of the nature of this group of conditions and gives an impression about the treatment of patients with Duchenne muscular dystrophy that would be seriously misleading to the non-specialist. Notwithstanding these irritations, the very high number and quality of the illustrations raise this volume above the ordinary and the reader is drawn through the text all the more easily.

Overall, I think that the authors have succeeded in their aim to provide two books that attempt to condense the neurosciences field into a concise and integrated format. From whichever angle one approaches them, the volumes as a whole are compelling reading and contain many useful factual nuggets. They appear to be particularly suitable for those preparing for neurosurgical examinations and are certainly a useful read for neurologists in training. Most of the underlying concepts will endure and can be augmented by the reader as developments ensue. They are of good quality production and reasonably priced.

Fiona Norwood 